\title{
Calidad de movimiento en adolescentes practicantes y no practicantes de Gimnasia Acrobática mediante la batería Funtional Movement Screen Quality of movement in Acrobatic Gymnastics teenagers and non-practitioners using the Funtional Movement Screen battery \\ Mercedes Vernetta Santana, Alicia Salas M orillas, Eva M a Peláez-Barrios, Jesús López-Bedoya \\ Universidad de Granada (España)
}

\begin{abstract}
Resumen. 0 bjetivo:Val orar la calidad de movimiento aplicando el Functional M ovement Screen (FMS) en adolescentes de 12 a 17 años practicantes o no de GimnasiaAcrobática (GA). M étodo: Estudio descriptivo, comparativo y transversal donde participaron 41 adolescentes (20 practicantes de GA y 21 no practicantes). Se usó la bateríaFM S, compuesta por siete pruebas: sentadilla profunda, paso de valla, estocada en línea, movilidad de hombro, elevación activa de la pierna recta, estabilidad del tronco en flexiones, estabilidad rotatoria del tronco. Resultados: se observó una relación estadísticamente significativade signo positivo a través del estadístico $R$ de Spearman, entre la puntuación global del FMS y la práctica de GA $(p=.023)$. Las gimnastas presentan mejor calidad de movimiento que las no practicantes, con diferencias estadísticamente significativas, puntuando mejor en prácticamente todas las pruebas. Conclusión: se corrobora una relación entre la práctica de GA y una mayor puntuación del FMS.
\end{abstract}

Palabras Clave: movimiento funcional, FMS, competencia motriz, gimnastas, adolescentes.

\begin{abstract}
To assess the quality of movement by applying the Functional Movement Screen (FM S) to teenagers aged 12 to 17, whether or not they practice Acrobatic Gymnastics (AG). M ethods: Descriptive, comparative and crosssectional study in which 41 teenagers (20AG practitioners and 21 non-practitioners) participated. The FM S battery was used, consisting of seven tests: deep squat, fence step, line lunge, shoulder mobility, active straight leg lift, trunk stability in bending, trunk rotation stability. Results: a statistically significant positive relationship was observed through Spearman's R statistic, between the overall FMS score and the AG practice $(p=.023)$. Gymnasts presented better quality of movement than nongymnastics practitioners, with statistically significant differences, scoring better in practically all tests. Conclusion: arelationship between AG practice and a higher FMS score is corroborated.
\end{abstract}

Keywords: functional movement, FMS, motor competition, gymnasts, adolescents.

\section{Introducción}

La práctica de actividad física (AF) en la adolescencia juega un papel importante a la hora del desarrollo de los ámbitos de la conducta cognitivo, afectivo, social y motriz.

Diversos estudios confirman la existencia de una relación favorable entre el desempeño motor en patrones de movimientos básicos con el nivel de AF y la participación en actividades físicas organizadas (Holfelder \& Schott, 2014). En base a esto suponemos que los alumnos que participen en actividades físicas extraescolares tendrán más desarrollados los patrones de movimiento fundamentales frente aquellos que no practican ninguna.

Una de las baterías para valorar la calidad del movi-

Fecha recepción: 04-12-20. Fecha de aceptación: 19-02-21

Alicia Salas Morillas

aliciasalasmorillas@gmail.com miento global, es el Functional Movement Screen (FMS), compuesta por siete pruebas específicas de movimiento relacionados con la movilidad, estabilidad y equilibrio que pueden detectar la existencia o no de posibles desequilibrios bilaterales de cada segmento corporal involucrado (Cook, Burton \& Hogenboom, 2006a; 2006b), haciendo de esta batería un potencial predictor de lesiones (Bardenett et al., 2015).

Se trata de un método simple y cuantificable de eva luación de patrones fundamentales de movimiento, capaz de establecer perfiles y comparaciones entre individuos del mismo deporte 0 de diferentes deportes (Fernández-Pino et al., 2017; Villalobos-Samaniego et al., 2020). A demás de ser una batería formada por pruebas sencillas, que no requiere mucho tiempo y espacio, y que necesita poco material y fácil de encontrar, su fiabilidad es excelente según el metanálisis realizado por Bonazza, Smuin, 0 nks, Silvis, \& Dhawan (2017).

La GimnasiaAcrobática (GA) es una disciplina gimnásticaintegrada en laFederación Internacional de Gim- 
nasia desde 1999 y consolidada como deporte de competición a nivel internacional. Es un deporte eminentemente cooperativo, donde la conjunción de los movimientos técnicos y corporales unidos y sincronizados a un soporte musical, constituye la esencia de esta disciplina deportiva, de ahí, que el componente artísticoestético tenga un lugar clave en esta nueva modalidad (Vernetta, Gutiérrez \&López-Bedoya, 2008).

Se trata de un deporte complejo con una elevada dificultad motriz, pero que, si se aprende de manera progresiva, es altamente atractivo para los adolescentes, ya que experimentan su gran progreso de manera constante. Desde el ámbito de la Educación Física (EF) el profesorado puede fomentar la adherencia a él, planteando intervenciones en su propia clase, mediante la organización de unidades didácticas de deportes gimnásticos con adaptaciones educativas como puede ser el Acrosport denominación de la GA en el ámbito escolar (Vernetta, López-Bedoya \& Panadero, 2007).

No hay constancia del uso de dicha batería en deportes gimnásticos, no encontrándose ninguno realizado en GA. Además, su aplicación en el ámbito escolar está aún poco extendida(Anderson, Neumann \& Bliven, 2015; García-Jaén, Pérez, Cortell-Tormo, Valero \& Anta, 2018; Vernetta, De 0 rbe, Peláez \& López-Bedoya, 2020a). De aquí que el objetivo de nuestro estudio fue evaluar la calidad de movimiento global en función de los patrones de movimiento fundamentales aplicando el Funtional Movement Screen en adolescentes practicantes de GA frente a no practicantes.

\section{Material y método}

\section{Participantes}

Estudio descriptivo de corte transversal y comparativo con la participación voluntaria de 41 adolescentes femeninas de Granada, cuya selección fue intencional. Del total de la muestra, 20 realizaba GA a nivel competitivo autonómico y nacional y 21 de ellas no practicaban ningún deporte en horario extraescolar. El total de la muestra se enmarca entre los 12 y 17 años. La muestra de gimnastas tenía una experiencia de entrenamiento promedio de $3 \pm 1$ años y entrenaban de 3 a 4 días a la semana con una media de $3 \pm 1$ h por sesión.

Para ser incluidos en el estudio, los participantes no debían de tener ningún tipo de lesión músculoesquelética, ni presentar problemas vestibulares, visua les o de equilibrio, que le impidiese ejecutar las diferentes pruebas requeridas en el FMS, se averiguó pa sando un cuestionario antes de comenzar.
El estudio se realizó de acuerdo con la Declaración de Helsinki para la experimentación humana y fue aprobado por el comité de ética de la U niversidad de Gra nada, número 1.011.

\section{Instrumento y materiales}

Para analizar la variable de calidad de movimiento se usó la batería FMS, creado por Gray Cook y Lee Burton en 1998, cuyostres objetivosfundamentales son; evaluar los patrones básicos del movimiento, detectar asimetrías y valorar el control motor (Dorrel, Long, Shaffer, \& M yer, 2015). Esta batería está compuesta por siete pruebas: sentadilla profunda, paso de valla, estoca da en línea, movilidad de hombro, elevación activa de la pierna recta, estabilidad del tronco en flexiones, esta bilidad rotatoria del tronco.

Se utilizaron los siguientes materiales: una pica de $1.22 \mathrm{~m}$; dos picas de $61 \mathrm{~m}$; un tablón de madera de $2 \mathrm{x}$ $6 \mathrm{~cm}$; una cinta métrica inelástica y dos cámaras de alta definición con tecnología de grabación 4K para grabar la ejecución de los ejercicios.

\section{Procedimiento}

En primer lugar, antes de la toma de datos se obtuvo la autorización del centro escolar donde pertenecían las participantes no practicantes y del club donde practicaban las gimnastas tras exponer los objetivos estudio, así como las pruebas a realizar. Igualmente, se contó con la firma del consentimiento informado de los pa dres 0 tutores de las participantes implicadas.

Las evaluaciones se llevaron a cabo de manera individual, realizándose tres veces cada una de las pruebas que componen la batería del FMS siguiendo el orden y loscriterios de puntuación establecidos por Cook (2010).

En todas las pruebas a excepción de la sentadilla profunda y la estabilidad del tronco en flexiones, se realizó una evaluación bilateral paradetectar posiblesasimetrías.

Las participantes realizaron un pequeño calentamiento dinámico antes de completar las pruebas de la batería. Se les facil itó instrucciones verbales de cada ejercicio siguiendo las pautas de descripción propuestas por Cook (2010), cada participante tenía tres oportunida des de ejecución para realizar un intento correcto.

La puntuación máxima que se puede obtener en la batería en general es de 21 puntos, siendo tres puntos la puntuación máxima por prueba. Los puntos se otorgan de acuerdo a los siguientes criterios: 3 puntos si los escolares 0 gimnastas practicantes eran capaces de realizar el movimiento, cumpliendo todos los criterios esta blecidos sin necesidad de aplicar una compensación; 2 
puntos si consigue realizar el movimiento con necesidad de una o más compensaciones; 1 punto si no es ca paz de completar el movimiento y se le otorgó 0 puntos si no podía completar el ejercicio o lo completa, pero siente dolor al realizarlo (Cook et al., 2006a).

En las pruebas bilaterales se tomó la puntuación inferior de las dos para el cálculo de la puntuación total de la batería. Si el sujeto presenta dolor durante el movimiento, automáticamente obtiene una puntuación de cero en dicha prueba, independientemente de la puntuación obtenida anteriormente (Cook, et al., 2006a).

La ejecución de cada participante se grabó digitalmente en vídeo desde diferentes planos de movimiento (frontal y lateral) y posteriormente fue analiza da conjuntamente por dos evaluadores con experiencia previa en el uso del FMS (Anderson et al., 2015).

\section{Análisis estadístico}

Los datos descriptivos en cada una de las pruebas de la batería, se muestran con la media y desviación típica. Asimismo, se calcularon la frecuencia y los val ores porcentuales de todas las pruebas, diferenciando el lado corporal en aquellas pruebas bilaterales. Como las puntuaciones obtenidas en las distintas pruebas en función de la variable práctica de GA no seguían una distribución normal en ningún caso, se optó por realizar un aná lisis no paramétrico. Al tratarse de muestras independientes y variable no normal se empleó la prueba $U$ de Mann W hitney. Finalmente, se hizo el análisis correlacional a través del estadístico R de Spearman, para conocer si la puntuación global de la batería se relaciona con la práctica de GA. Todos Ios anál isis estadísticos fueron realizados utilizando el programa SPSS en su versión 22.0 (SPSS Inc., Chicago IL, USA) y el nivel de significación fue de $p<.05$.

\section{Resultados}

LaTabla 1 muestra la estadística descriptiva (media y desviación típica) y las diferencias de las puntuaciones obtenidas en cada una de las pruebas de FMS, en función delosdosgrupos (practicantesy no practicantes deGA). Los resultados revelaron que las practicantes de GA obtuvieron mayores puntuaciones con diferencias significativas en cinco pruebas excepto en la movilidad de hombro, siendo las no practicantes las que obtuvieron mayor puntuación en el lado derecho. La prueba estabilidad rotatoria del tronco fue la única en la que no se apreció diferencias entre ambos grupos.

\begin{tabular}{|c|c|c|c|}
\hline Prueba test FMS & $\begin{array}{c}\text { No practicantes } \\
(\mathrm{n}=21)\end{array}$ & $\begin{array}{c}\text { PracticantesGA } \\
(\mathrm{n}=20)\end{array}$ & $p$ \\
\hline Sentadilla profunda & $2.1 \pm .3$ & $2.8 \pm .3$ & $.000^{*}$ \\
\hline Paso de valla (dcha) & $2.9 \pm .2$ & $2.9 \pm .2$ & $.972^{*}$ \\
\hline Paso de valla (izq) & $2.7 \pm .4$ & $2.9 \pm .2$ & .092 \\
\hline Estocada en línea (dcha) & $2.0 \pm .4$ & $2.8 \pm .3$ & $.000^{* *}$ \\
\hline Estocada en línea (izq) & $1.6 \pm .4$ & $2.9 \pm .3$ & $.000^{* *}$ \\
\hline Movilidad de hombro (dcha) & $2.7 \pm .4$ & $2.2 \pm .7$ & $.036^{*}$ \\
\hline Movilidad de hombro (izq) & $2.3 \pm .4$ & $2 \pm .6$ & .054 \\
\hline Elevación activa de la pierna recta (dcha) & $2.2 \pm .5$ & $3 \pm 0$ & $.000 *$ \\
\hline Elevación activa de la pierna recta (izq) & $1.9 \pm .5$ & $3 \pm 0$ & $.000^{*}$ \\
\hline Estabilidad del tronco en flexiones & $1.9 \pm .7$ & $2.6 \pm .5$ & $.002^{* *}$ \\
\hline Estabilidad rotatoria tronco (dcha) & $2.7 \pm .4$ & $2.7 \pm .5$ & .600 \\
\hline Estabilidad rotatoria tronco (izq) & $2.5 \pm .5$ & $2.7 \pm .5$ & .162 \\
\hline Puntuación total en la batería FMS & $15.2 \pm 1.5$ & $18.7 \pm 1.3$ & $.000^{* *}$ \\
\hline
\end{tabular}

En laTabla 2 se observa la frecuencia y el porcentaje obtenido en las diferentes pruebas. En el caso de las practicantes de GA, se destacó que en todas las pruebas la gran mayoría alcanzaron la máxima puntuación de 3. La puntuación nunca fue cero para ninguna de las participantes. Por otro lado, en las que no practican GA, la única prueba en la que ninguna al canzó la máxima puntuación fue en la estocada en línea por el lado corporal izquierdo.

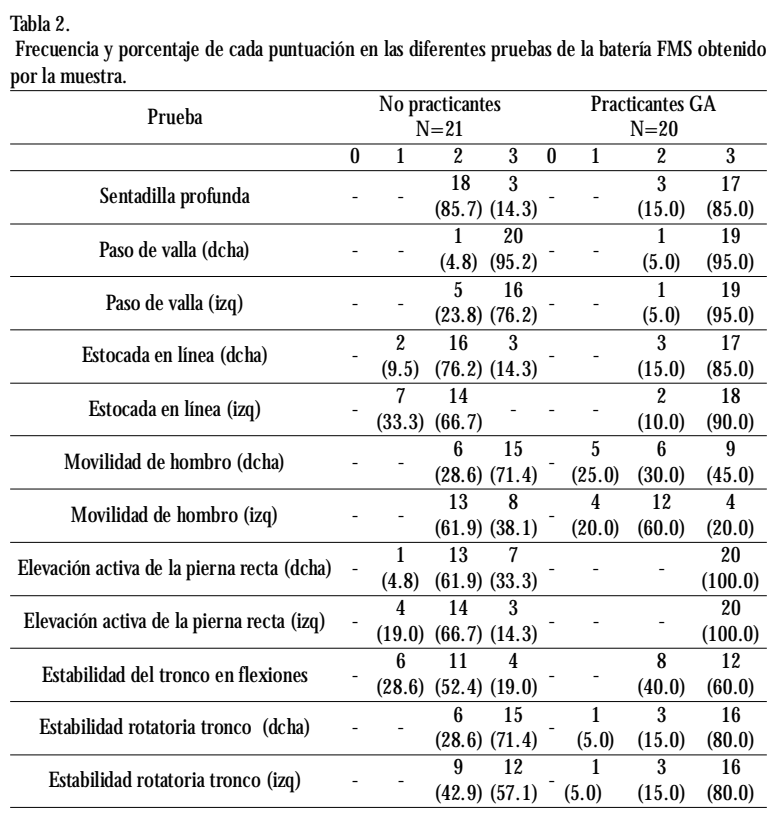

LaTabla 3, muestran la clasificación de la muestra en función de la puntuación total obtenida en la batería. Se observó como la mayor parte de las participantes alcanzaron un nivel aceptable de calidad de movimiento, siendo superior en las practicantes de GA.

\begin{tabular}{|c|c|c|}
\hline Puntuación FMS & $\begin{array}{c}\text { No practicantes } \\
\mathrm{N}(\%)\end{array}$ & $\begin{array}{c}\text { Practicantes GA } \\
\mathrm{N}(\%)\end{array}$ \\
\hline$\leq 14$ & $6(28.6 \%)$ & $0(0)$ \\
\hline $15-20$ & $15(71.4 \%)$ & $18(90 \%)$ \\
\hline 21 & $0(0)$ & $2(10 \%)$ \\
\hline
\end{tabular}


El estadístico U de Mann W hitney, para muestras independientes arrojó diferencias estadísticamente significativas entre los valores del FMS en relación con la práctica de $\mathrm{GA}(U=18 ; p=.000)$. Las practicantes de GA tuvieron rangos promedio mayores que aquellas que no la practicaban, siendo éstos 30.60 y 11.86 respectivamente (Figura 1 ).

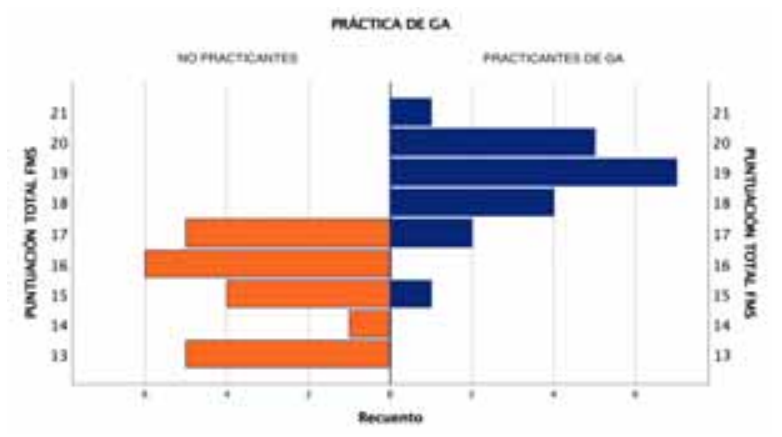

Figura 1. Diferencias mostradas por la prueba U de MannW hitney para muestras independientes. Puntuación global de la batería FMS en relación a la práctica de GA.

Finalmente, en la Tabla 4, el estadístico $R$ de Spearman mostró una relación estadísticamente significativa de signo positivo entre las variables puntuación global del FM S y la práctica de GA $(p=.023)$.

\begin{tabular}{|c|c|c|c|c|}
\hline & & & $\begin{array}{l}\text { Prácticade } \\
\text { GA }\end{array}$ & $\begin{array}{l}\text { Puntuación total } \\
\text { FMS }\end{array}$ \\
\hline $\begin{array}{l}\text { E. Rho de } \\
\text { Spearman }\end{array}$ & Práctica de GA & $\begin{array}{l}\text { Coeficiente de correlacióm } \\
\text { Sig (bilateral) }\end{array}$ & 1.000 & $\begin{array}{c}.799 * * \\
.023\end{array}$ \\
\hline & $\begin{array}{l}\text { Puntuación } \\
\text { total FMS }\end{array}$ & $\begin{array}{l}\text { Coeficiente de correlacióm } \\
\text { Sig (bilateral) }\end{array}$ & $\begin{array}{l}0.330 \\
0.023\end{array}$ & 1.000 \\
\hline
\end{tabular}

\section{Discusión}

El objetivo principal del estudio fue valorar la calidad de movimiento a través del FMS en a dolescentes de 12 a 17 años en función de si practican o no GA. Como resultados principales cabe destacar niveles aceptables de FMS en la muestra total, encontrándose mejor calidad de movimiento en las practicantes de GA, con mejores puntuaciones en la mayoría de las pruebas. De igual manera, existió una asociación entre los valores más elevados del FMS con la práctica de GA.

Con respecto a la puntuación gl obal del FMS, ninguna de las practicantes de GA, puntuó por debajo o igual alapuntuación de 14 puntos, correspondiente aun nivel bajo 0 de riesgo de lesión, no siendo así para las no practicantes, donde seis de ellas, obtuvieron un nivel bajo. La mayoría de la muestra, tanto practicantes de GA como no practicantes, obtuvieron puntuaciones, entre 15-20 puntos correspondiente a un nivel aceptable. Esto apoya los resultados obtenidos por GarcíaPinillos et al.
(2019), donde los participantes de mayor edad (adolescentes) obtuvieron mejores resultados en la puntuación total de la batería.

Los mejores resultados de las practicantes de GA en relación a las no practicantes, coinciden con los hallados por Kryak et al. (2019) y Vernetta, De O rbe $\&$ Salas (2020b), donde los adolescentes que practicaban actividades deportivas obtuvieron mejores resultados, posiblemente debido a su mayor desarrollo motriz genera do por la práctica deAF. Igualmente, corrobora en parte la posible relación existente entre el desempeño motor en patrones de movimientos básicos con la participación en actividades físicas organizadas (Holfelder $\&$ Schott, 2014). Destacar que ninguna de las adolescentes no practicantes alcanzó la nota máxima de 21 puntos, siendo lograda por dos practicantes de GA. Teniendo en cuenta que el movimiento funcional se puede mejorar con programas de entrenamiento, se puede suponer que los entrenamientos a los que están sometidas las gimnastas de acrobática tienen un efecto significativo en los resultados más óptimos del FMS (Peate, Bates, Lunda, Francis, \& Bellamy, 2007).

En relación a la movilidad articular, las practicantes presentan mejor puntuación en la elevación de piernas donde todas obtienen una puntuación máxima de 3 puntos en ambos lados. Estos resultados son esperables, debido a la importancia de la flexibilidad considerada capacidad preponderante en los deportes gimnásticos, siendo además la flexibilidad de la articulación de la cadera-extremidad inferior fundamental (Sands, M cN eal, Stone, Russell \& Jemni, 2006; D onti, Bogdanis, Kritikou, Donti, \& Theodorakou, 2016). Sin embargo, sorprende que en la movilidad de hombro las no practicantes obtienen mayor puntuación (donde ninguna obtuvo la puntuación de 1), frente a las practicantes donde el $25 \%$ y el $20 \%$ (derecha e izquierda) obtuvieron 1 punto. La amplitud de movimiento en los hombros, tiene una gran importancia a nivel técnico en esta disciplina, tanto en el trabajo mano a mano de portores, como en el trabajo de apoyos invertidos de los ágiles. El estudio de Irurtia, Busquets, Carrasco, Ferrer, \& Marina (2010) indican que la movilidad de hombro en los niveles más elevados de competición (en gimnasia artística) tiene una relación negativa con el rendimiento (a ma yor rendimiento, menor es el rango de movimiento en hombros), podría tener relación con las gimnastas que puntuaron más bajo en esta prueba, ya que eran las que mejor nivel de rendimiento tenían en competición. Se podría intuir por los bajos resultados de esta prueba, que un mayor desarrollo de la masa muscular, con pre- 
dominio de las extremidades superiores tanto por parte de las ágiles como de las portoras y sobre todo en el brazo dominante, justificaría su peor puntuación en esta prueba. No obstante, la información bilateral de esta prueba, puede aportar pautas funcional es de interés que deban ser tenidas en cuenta en el entrenamiento de los gimnastasy en la prevención de lesiones. Si bien la prueba Shoulder Flexibility incluida en el FMS, forma parte también de baterías funcionales muy reconocidas como el Fitnessgram en USA, Plowman, Sterling, Corbin, Meredith, Welk \& Morow (2006), ésta ha sido escasa mente utilizada en gimnastas y, por tanto, se requieren mas estudios para comprobar su validez y aplicación en un tipo de población tan específica. Q uizás la utilización adicional de otros test que han mostrado su validez y fiabilidad con gimnastas femeninas como el Shoulder Flexibility Test, para medir el rango de movimiento activo en laflexión del hombro, Sleeper, Kenion \& C asey (2012), podrían constatar el grado de correlación entre ambas pruebasy deestaformatener una evidenciamayor, para establecer determinadas acciones correctoras en términosfuncionalesy de entrenamiento para gimnastas de acrobática. En cuanto a los resultados de las no practicantes son similares a los deVernetta et al. (2020a), en un grupo de chicas escolares de secundaria.

En la prueba de sentadilla profunda, en las no practicantes la mayoría (85.7\%) obtiene la puntuación de 2, seguido de un $14.3 \%$ que obtienen 3 puntos. En cambio, en las practicantes de GA el $85 \%$ obtienen 3 puntos frente aun $15 \%$ que puntuaron 2 . Estos datos nos muestran que no existen grandes dificultades en la realiza ción de la prueba, siendo similares a los deVernetta et al. (2020b), con una muestra de practicantes de baloncesto y no practicantes. En ambos estudios, los datos son mayores en las deportistas tanto de GA como de bal oncesto. Esto puede ser posible debido a que la sentadilla representael movimiento por excelenciademusculación de los miembros inferiores en cualquier plan de entrenamiento, que recluta gran número de grupos musculares (cuádriceps, gemelos, glúteos, abdomen y espalda), desarrollando no solo la fuerza en los mismos, lo que permite mayor eficacia en acciones de saltar, empujar y contraer, sino también ayuda a corregir la postura y tener un mejor desarrollo motriz (M edina, 2003; Rippetoe, 2012). No obstante, en este estudio, las practicantes de GA obtienen datos ligeramente superiores a las de baloncesto. Puede deberse a la amplitud de movimiento, siendo esta mayor en gimnastas, ya que se trata de una prueba de gran importancia en deportes gimnásticos en general y en la GA en particular, siendo muy utilizada sobre todo por los portores como movimiento base para pasar al suelo (transiciones) en los elementos cooperativos con el agil, o para propulsarlos en los elementos dinámicos (Vernetta, Jiménez \& LópezBedoya, 2007).

Por otra parte, al ser una prueba de sentadilla sosteniendo la pica con los brazos extendidos sobre la cabeza, exige en el participante posibles ajustes compensatorios en la activación del centro de masa y una mejora de la capacidad de respuestas de los múscul os posturales (G lass \& Albert, 2018). Se ha documentado que la práctica de disciplinas gimnásticas desde los primeros años de vida mejora el desarrollo del control postural y una mayor estabilidad postural (García, Barela,Viana\& Barela, 2011; 0 pala-Berdzik, GBowacka \& Juras, 2021). Este aspecto está muy desarrollado en estos gimnastas ya que el control postural juega un papel importante en este deporte sobre todo en las acciones con cambios de posiciones (motions) con el ágil sobre su cabeza ya que las posibles perturbaciones provocadas por los movimientos del mismo, exige un gran control postural independientemente de la posición que adopte.

Con respecto al equilibrio, la prueba de paso de vaIla fue la que mejor resultados obtuvo para ambos grupos, destacando mejor puntuación en las practicantes, donde el 95\% de ellas lograron los 3 puntos máximos en ambos lados. En las no practicantes se observa una pequeña descompensación en el lado izquierdo ya que el $95.2 \%$ obtuvieron 3 puntos en el lado derecho y un $76.2 \%$ en el lado izquierdo. En la prueba de estocada en línea junto a la estabilidad de tronco en flexiones, los resultados son similares a los de Bullok et al. (2017), existiendo una gran diferencia entre practicantes de GA y no practicantes. Destacar que, en la estocada en línea por el lado izquierdo, es la única prueba donde ninguna de las chicas no practicantes consigue alcanzar 3 puntos. Con respecto a las deficiencias observadas en esta prueba paralas no practicantes, autores como Cook (2001) y O kada, Huxel, \& N esser (2011) aconsejan un trabajo de la musculatura con programas de acondicionamiento y fuerza para el desarrollo de una mejor calidad en estos patrones, siendo el uso del FMS muy importante para identificar estos desbalances (Lisman, Hildebrand, Nadelen, \& Leppert, 2019).

En el caso a la prueba de estabilidad rotatoria de tronco, en general las chicas practicantes de GA obtienen en mayor porcentaje 3 puntos, estos datos no son coincidentes con Anderson et al. (2015) y Abraham, Sannsai \& Nair (2015), donde las practicantes de al guna AF organizadapresentan puntuacionesinferiores. En esta 
disciplina el control postural juega un papel importante, por lo que se trabaja desde sus inicios, ya que es totalmente necesario para la realización de cualquier gesto técnico. Con lo cual, el beneficio del entrenamiento neuromuscular en estas gimnastas puede ser una ganancia en las puntuaciones en estas pruebas de FM S (Portas, Parkin, Roberts, \& Batterham, 2019).

En relación a posibles asimetrías, el grupo de practicantes presentan puntuaciones muy similares en ambos lados, excepto en la movilidad de hombros donde si se pueden observar diferencias, siendo el lado derecho el predominante. En el grupo de no practicantes no existen grandes diferencias entre los dos lados, pero si es superior la diferencia con respecto a las practicantes, siendo la prueba de estocada en línea la que mayor diferencia presenta.

Ninguna gimnasta presentó asimetría en tres de las cinco pruebas: paso de valla, elevación activa de la pierna recta y estabilidad rotatoria tronco, con ligerísima asimetría en las dos restantes, no siendo así en las no practicantes, quienes presentaron asimetría en todas las pruebas. Es posible que esta mayor simetría en las gimnastas, se deba a las características motoras de la GA. Diferentes estudios han planteado que la práctica y la repetición continuada de tareas específicas de equilibrio en gimnastas conllevan una mejora del control postural en condiciones estáticas (Gautier, Thouvareca \& Larue, 2008; Calavalle et al., 2008). Estas tareas forman parte habitual de las rutinas de entrenamiento de la GA, así como de otras disciplinas gimnásticas que incluyen acrobacias entre sus elementos técnicos. Las pruebas bilaterales del paso de valla y estabilidad rotatoria del tronco al tener una estrecha base de sustentación, exigen un control dinámico de la pelvis y del core en relación a los músculos involucrados en la antirrotación y desplazamientos laterales. En definitiva, el control postural es un aspecto clave en los entrenamientos de estos gimnastas para la ejecucion correcta de diferentes elementos, ya que tanto portores como agiles deben dominar y mantener una adecuada posicion corporal para ejecutar de manera correcta y segura las diferentestecnicas de elevacion o de ascenso de un cuerpo sobre otro, así como el estacionamiento del mismo (Vernetta et al. 2007). Con lo cual nuestros resultados sugieren y apoyan la relación entre la estabilidad del core y la ejecución exitosa y simétrica de los patrones funcionales en estos gimnastas (0 kada et al.2011).

Por otro lado, aunque somos conscientes que en la GA como en la mayoría de los deportes gimnásticos se da preferencia al desarrollo de un lado del cuerpo, so- metiendo a las gimnastas a la repetición de los movimientos específicos en un solo lado, sin embargo, se puede destacar que este deporte al ser cooperativo por excelencia, los gimnastas en general, aunque tengan un predomino de lateralidad en un lado en muchas ocasiones utilizan los dos para sincronizar mejor sus acciones con su compañero.

Por último, se mostró una relación positiva entre los resultados globales del FMS y la practica de GA. Esto coincide con estudios que relacionan la práctica de AF y deportiva con la calidad de las pruebas del FMS (Bürgi etal. , 2011; J aakkola\&W ashington, 2013; Vernetta et al., 2020b).

En GA el nivel de ejecución técnica que exigen los elementos es muy alto, de ahí la interdependencia con la calidad de movimiento de las pruebas de FMS, lo cual replica lo indicado por Starosta et al. (2006) quienes realizaron un estudio con patinadores artísticos y encontraron que un mayor nivel de habilidad en el deporte se asocia con una mayor precisión de desempeño motriz o realización de movimientos específicos.

Finalmente destacar como limitaciones, la falta de estudios encontrados en los que se utilice el FMS para evaluar muestras en deportes gimnásticos o de carácter estético, con la cual ha sido prácticamente imposible comparar los resultados de esta muestra de practicantes. Igualmente, el tamaño reducido de la muestra, así como, que todas las gimnastas pertenezcan a un mismo club y con un nivel competitivo similar. 0 tra limitación de nuestro estudio fue no haber medido parámetros antropométricos en los adolescentes estudiados, ya que en la realización de las pruebas del FMS puede tener ciertainfluenciala constitución morfológica del ejecutante evaluado, (Perry \& Kochle, 2013). En futuros trabajos sería interesante ampliar la muestra a diferentes categorías, nivel competitivo e incluir ambos géneros.

\section{Conclusiones}

- Las adolescentes practicantes de GA presentan mejor calidad de movimiento con diferencias estadísticamente significativas frente a las no practicantes, existiendo una relación de signo positiva entre la práctica de GA y la puntuación total del FMS.

- Ninguna de las practicantes se encuentra en situación de riesgo, obteniendo todas un nivel aceptableu óptimo de calidad de movimiento, no siendo así en las no practicantes.

- Las gimnastas puntúan significativamente mejor 
en el test de sentadilla profunda, paso de valla, estocada en línea (ambos lados), elevación activa de la pierna recta (ambos lados) y estabilidad rotatoria tronco, no presentando asimetrías severas.

A nivel global, indicar que los resultados de las puntuaciones obtenidas en el FMS para adolescentes deben interpretarse con precaución, como indica el estudio de Mokha et al. (2016), en población adultay se debe prestar atención a las asimetrías identificadas durante la eva luación como un predictor de riesgo de lesiones futuras tanto en la población de no practicantes, como en las gimnastas de cara a una mayor especialización, ya que supondrá un incremento de la intensidad en sus entrenamientos. Como aplicación práctica, los entrenadores de esta disciplina gimnástica pueden integrar el FMS dentro de su batería de monitorización como una herramienta de detección de posibles desequilibrios funcionales en sus gimnastas por su bajo coste y efectividad en el tiempo.

\section{Referencias}

Abraham, A., Sannasi, R. \& Nair, R. (2015). Normative values for the functional movement screentm in adolescent school aged children. International journal of sports physical therapy, 10(1), 29.

Anderson, B. E., Neumann, M. L. \& Bliven, K. C. H. (2015). Functional movement screen differences between male and female secondary school athletes. The Journal of Strength \& Conditioning Research, 29(4), 1098-1106.

Bardenett, S. M., Micca, J. J., DeN oyelles, J. T., Miller, S. D., Jenk, D. T. \& Brooks, G. S. (2015). Functional M ovement Screen normative values and validity in high school athletes: can the FMS ${ }^{\mathrm{TM}}$ be used asa predictor of injury? International journal of sports physical therapy, 10(3), 303.

Bonazza, N. A., Smuin, D., Onks, C. A., Silvis, M. L. \& Dhawan, A. (2017). Reliability, validity, and injury predictive value of the functional movement screen: a systematic review and metazanalysis. TheAmerican Journal of Sports Medicine, 45(3), 725-732.

Bürgi, F., Meyer, U., Granacher, U., Schindler, C., Marques-Vidal, P., Kriemler, S., et al. (2011) Relationship of physical activity with motor skills, aerobic fitness and bodyfat in preschool children: a cross-sectional and longitudinal study (Ballabeina). Int] O bes, 35(7):937e944. http:/ / dx. doi.org/ 10.1038/ ijo.2011.54.42.

Calavalle, A.R., Sisti, D., Rocchi, M.B.L., Panebianco, R., del Sal, M. \& Stocchi,V. (2008) Postural trias: Expertise in rhythmic gymnastics increases control in lateral directions. Eur J Appl Physiol., 104 (4), pp. 643

Cook, G. (2001) Baseline sports-fitness testing. Champaign, IL: Human Kinetics Inc, 19-47.38.

Cook, G. (2010) M ovement: Functional movement systems Screening, assesment, corrective srategies. Book Baby.

Cook, G., Burton, L. \& Hoogenboom, B. (2006a) Preparticipation screening: the use of fundamental movements as an assessment of function - part 1. N Am J Sports PhysTher. 1(2):62.

Cook, G., Burton, L. \& Hoogenboom, B. (2006b) Preparticipation screening: the use of fundamental movements as an assessment of function - part 2. N Am J Sports PhysT her. 1(3):132-9.

Donti, O., Bogdanis, G. C., Kritikou, M., Donti, A. \& Theodorzkou, K. (2016). The relative contribution of physical fitness to the technical execution score in youth rhythmic gymnastics. Journal of Human Kinetics, 50(2), 143-152. https:/ / doi.org/ 10.1515/ hukin-2015-0183

Dorrel, B. S., Long, T., Shaffer, S. \& Myer, G. D. (2015) Evaluation of the Functional Movement Screen as an Injury Prediction Tool AmongActiveAdult Popultions. Sports Health, 7(6):53-59. https:/ / doi.org/ 10.1177/ 1941738115607445.

Fernández-Pino, J. A., Figueroa Contreras, D. E., GarcésMondría, F. I., Montalva Purcell, B., O livares, N. \& Alonso, R. (2017). Calidad demovimiento evaluado a través del Tes FMS en estudiantes de primer año de la carrera de Educación Física durante el año 2016 (Doctoral dissertation, Universidad Andrés Bello).

Gautier G., Thouvarecq R., Larue J. (2008). Influence of experience on postural control: Effect of expertise in gymnastics. J Mot Behav, 40 (5) pp. 400-408

Garcia, C., Barela, JA., Viana, AR., \& BarelaA.M. (2011). Influence of gymnastics training on the development of postural control. N eurosa Lett. 492, 29-32.

GarcíaJ aén, M. G., Pérez, S. S., Cortell-Tormo, J. M., Valero, A. F. \& Anta, R. C. (2018). Evaluación de los patrones de movimiento fundamentales en niños: comparación de género en escolares de Educación Primaria. Retos: nuevas tendencias en educación física, deporte y recreación, 34, 282-286.

GarcíaPinillos, F., Párraga-Montilla, J., Roche-Seruendo, L., Delgado-Floody, P., M artínez-Salazar, C.P., \& LatorreRomán, P. Á. (2019). Do age and sex influence on functional movement in school-age children? Retos nue vas tendencias en educación físca, deporte y recreación, (35), 97-100.

Glass, S.C. \& Albert, R.W. (2018). Compensatory muscle activation during unstable overhead squat using waterfilled training tube. Journal of Strenght and Conditioning 
Research, 32(5), 1230-1237

Holfelder, B. \& Schott, N. (2014). Relationship of fundamental movement skills and physical activity in children and adolescents: A systematic review. Sport Exerc Perform Psycho, 15 (4):382-391. https:/ / doi.org/ 10.1016/ j. psychsport. 2014.03.005

Irurtia, A., Busquets, A., Carrasco, M., Ferrer, B. \& Marina, M. (2010). Control de la flexibilidad en jóvenes gimnastas de competición mediante el método trigonométrico: un año de seguimiento. Apunts M ediana del'Esport, 45(168), 235-242. https./ / doi.org/ 10.1016/ j.apunts.2010.05.003

Jazkkola,T. \&Washington,T. (2013)Therelationship between fundamentalmovement skills and self-reported physical activity during Finnish junior highschool. Phys Educ Sport Pedagogy., 18(5):492e505. http:/ / dx. doi.org/ 10.1080/ 17408989.2012.690386

Lisman, P., Hildebrand, E., Nadelen, M. \& Leppert, K. (2019). Association of Functional M ovement Screen and Y-Balance Test Scores with Injury in High School Athletes. Journal of trength and conditioning research. DO I: https:/ / doi.org/ 10.1519/ JSC.0000000000003082

Medina, E. (2003). Actividad Física y Salud Integral. Barcelona: Paidotribo.

Mokha, M. ., Sprague, P.A. \& G đtens, D. R. (2016). Predicting musculoskeletal injury in national collegiate athletic association division II athletes from asymmetries and individual-test versus composite functional movement screen scores Journal of Athletic Training, 51, $276 \mathrm{e} 282$.

O kada, T., Huxel, K. C.\& Nesser, T.W. (2011) Relationship between core stability,functional movement, and performance. J Strength Cond Res, 25(1):252-261.https:/ / doi.org/ 10.1519/ JSC.0b013e3181b22b3e.

O pala-Berdzik,A., GBowacka, M. \& Juras, G. (2021). Postural sway in young female artistic and acrobatic gymnasts according to training experience and anthropometric characteristics. BMC Sports Science, Medicine and Rehabilitation, 13(11), 2-11. https:/ / doi.org/ 10.1186/ s13102-021-00236-w

Peate, W. F., Bđes, G., Lunda, K., Francis, S., \& Bellamy, K. (2007). Corestrength: anew model for injury prediction and prevention. Journal of Occupational Medicine and Toxicology, 2(3), 1-9.

Perry F.T. \& Koehle M.S. (2013). Normative data for the functional movement screen in middle-aged adults. J Strength Cond Res, 27(2):458-62.

Plowman, S.A, Sterling, C.L Corbin, C.B, Meredith, M.D, Welk G.J \& Morow J.R. (2006). The history of fitnessgram. Journal of Physical Adivity \& H ealth (Suppl.2), S5.S2O.
Portas, M. D., Parkin, G., Roberts, J. \& Batterham, A. M. (2016) Maturational effecton Functional Movement Screen $^{\text {TM }}$ score in adolescent soccer players. I SciM ed Sport, 19(10):854-858. https:/ / doi.org/ 10.1016/ j.jsams. 2015.12.001.

Rippetoe, M. (2011). Starting Strenght. The Aasgaard Company: USA tercera edición.

SandsWA, M cNeal JR, Stone MH, Russell EM \& Jemni M. (2006) Flexibility enhancement with vibration: acute and long-term. Med Sá Sports Exerc, 38:720-5.

Sleeper, M.D, Kenyon, L.K, Casey, E. (2012). Measuring fitness in female gymnasts: the gymnastics functional measurement tool. The International Journal of Sports Physical Therapy, 7, 2, pp 124-138.

Starosta,W. (2006). The concept of modern training in sport. Studies in Physical Culture and Tourism, 13 (2), 9-23.

Vernetta-Santana, M., deO rbe-M oreno, M., Pelæz-Barrios, E.M. \& Lopez-Bedoya, J. (2020a) Movement quality evaluation through the functional movement screen in 12- and 13-year-old secondary-school adolescents. JHSE, 15(4), 918-931 doi: https:/ / doi.org/ 10.14198/ jhse. 2020.154.18.

Vernetta, M., De O rbe, M. \& Salas, A. (2020b) Práctica extraescolar del baloncesto y calidad de movimiento en chicas adolescentes. Revista Iberoamericana de Ciencias de la Actividad Físca y el D eporte, 9(2) 75-93.

Vernetta, M., Jiménez, J. \& López-Bedoya, J. (2007). La utilización del registro de los tiempos de intervención delasaccionesmotrices en lagimnasiaacrobática. Revista Digital, 12, 110.

Vernetta, M., López, J. \& Gutiérrez, A. (2008). La creatividad de la gimnasia acrobática. EnA. Martínez \& P. Díaz (Coord.), Creatividad y deporte. Consideracionesteóricas einvestigaciones breves (pp. 133-156). Sevilla: Wanceulen.

Vernetta, M., Lopez Bedoya, J. \& Panadero, F. (2007). El Acrosport en la escuela. . (3a ed.) Barcelona: Inde.

Villalobos-Samaniego, C., Rivera-Sosa, J. M., RamosJimenez, A., Cervantes-Borunda, M. S., Lopez-Alonzo, S. J., \& Hernandez-Torres, R. P. (2020). Métodos de evaluación del equilibrio estático y dinámico en niños de 8 a 12 años (Evaluation methods of static and dynamic balance in children aged 8 to 12 years old). Retos, (37), 793-801. 